Volume 10, No.3, May - June 2021

International Journal of Science and Applied Information Technology

Available Online at http://www.warse.org/ijsait/static/pdf/file/ijsait011032021.pdf

https://doi.org/10.30534/ijsait/2021/011032021

\title{
Background Subtraction of an Indian Classical Dance Videos using Adaptive Temporal Averaging Method
}

\author{
1 Bhavana R.Maale, ${ }^{2}$ Dr.Suvarna.Nandyal \\ ${ }^{1}$ Visvesvaraya Technological University,India, sg.bhavana@ gmail.com \\ ${ }^{2}$ PDA College of Engineering,India, suvarna_nandyal@yahoo.com
}

\begin{abstract}
A slew of motion detection methods have been proposed in recent years. The background includes some constraints such as changes in illumination, shadow, cluttered the background, scene change and speed of dance between hand gestures and body gestures are different. One of the most basic methods for background subtraction is temporal averaging. We looked at a new adaptive temporal averaging approach in this paper. To identify moving objects in video sequences, an adaptive temporal averaging technique is used. Depending upon the speed of the technique we proposed a Gaussian distribution technique. Gaussian distribution done background subtraction depending upon active pixels it differentiates whether it is a background or foreground. The background model's update rate has been modified to be adaptive and determined by pixel difference .Our aim is to improve the method's F-measure by making it more adaptable to various scene scenarios. The experiment results are shown and evaluated. The proposed method and the original method's quality parameters are compared.
\end{abstract}

Key words: Motion Detection, Background Subtraction, Gaussian Distribution, The Adaptive temporal averaging method.

\section{INTRODUCTION}

Analysis and comprehension of video sequences is a growing area of study. The identification of moving objects in the scene is a first step in many applications in this area (video tracking, optical motion capture, multimedia applications). The separation of moving objects, referred to as the foreground, from static data, referred to as the background, is the basic operation required. Background
Subtraction is the most commonly used process. Obtaining a background image that does not contain any moving objects is the best way to model the background. The background is not always available in certain environments, but it can always be altered in crucial circumstances such as lighting changes or the addition or removal of items from the scene. As a result, the background representation model must be more adaptable and stable. Every modern video monitoring system includes motion detection.

In computer vision systems, background subtraction is a popular technique for identifying movement regions. It simply refers to the division of the current scene into two parts: the background and foreground .Using a constant model for the background is one of the easiest ways to enforce background subtraction. The moving regions are then distinguished from the background using this model. However, there are several drawbacks to this approach. It is incapable of dealing with changes in lighting, objects being shifted to or removed from the background, shadows, and repeated motion such as the tree leaves, among other things. More intelligent approaches to adapt the background model to the evolving world have been implemented to address at least some of these problems.

Intelligent control systems are capable of distinguishing between moving objects in the foreground and stationary objects in the background, in addition to detecting motion. In recent years, several of these motion detection methods have been proposed. The most widely used techniques for this separation are background subtraction methods [13].Statistical approaches such as Mixture of Gaussians [4-8], Kernel Density Estimation [9], and Eigen backgrounds [10] are more accurate and complex. 
Bhavana R.Maale et al., International Journal of Science and Advanced Information Technology, 10 (3), May - June 2021 , 16 - 19

The Temporal Averaging Method (TAM) [11-13] is a simple method for background subtraction that is the subject of this article.The original method is shown, and new versions of the algorithm are proposed in this paper. The background model is the first step in the original TAM process. It represents a portion of the scene that is relatively still. algorithm (3). We find the threshold $t$ adaptive to each pixel difference $d_{t_{(a, b)}}$, just as in the first approach

\section{OUR APPROACH}

When the scene illumination is constant, the original TAM approach is reasonably effective in estimating foregroundbackground. However, when there are sudden lighting changes or repeating backgrounds, such as waving trees, there are a lot of false positive pixels and the output parameter levels are lower. The original method's flaw is the slowness with which

it updates the background model determined by $\alpha$, (1). For all pixels in the current frame, the method assumes $\alpha$ is equal. When the background is changing rapidly, this is not the best choice. So, if the updating speed could be adjusted to each pixel difference $d_{t_{(a, b)}}$, the algorithm would respond faster to sudden light changes and repeated backgrounds. Furthermore, the above-mentioned solution would be insufficient in very noisy and complex background scenes. An adaptive threshold will be used to reduce the number of false positive pixels in the predicted scene. The threshold $\mathrm{T}$ is constant in the original algorithm (3). We find the threshold $t$ adaptive to each pixel difference $d_{t_{(a, b)}}$, just as in the first approach.

\section{ALGORITHM}

For every incoming image a new background model

$C_{t \square x, y \square}$ is estimated by:

$c_{t+1_{(a, b)}}=\alpha I_{t_{(a, b)}}+(1-\alpha) c_{t_{(a, b)}} \quad \mathrm{Eq}-1$

$I_{t_{(a, b)}}$ is the pixel value, $t$ is the image number, $(a, b)$ is the pixel location in the image and $\alpha$ is learning rate.

The first background is formed by the pixels that have least absolute difference value. Then the absolute difference is calculated by the following equation:

$$
d_{t_{(a, b)}}=\left|I_{t_{(a, b)}}-b_{t_{(a, b)}}\right| \quad \mathrm{Eq}-2
$$

The sum of absolute difference makes pixel by pixel comparison between two consecutive frames. The absolute difference are added up the pixels that have least sum of absolute difference value is the background. To handle variation in background such has color and illumination changes. The rule of updating $\alpha_{t_{(a, b)}}$ is given by:

$$
\alpha_{t+1_{(a, b)}}=\beta \cdot\left(\frac{c \cdot d_{t_{(a, b)}}}{n}\right)+(1-\beta) \cdot \alpha_{t_{(a, b)}} \quad \mathrm{Eq}-3
$$

$\beta$ is the adaptive learning rate updating $\alpha_{t+1_{(a, b)}}$, c value is given by the user, $\mathrm{n}$ is the dynamic range of the signal.

The foreground mask is estimated by the equation :

$$
N_{t_{(a, b)=}}=\left\{\begin{array}{l}
0, d_{t_{(a, b)}}<T 1_{t_{(a, b)}} \\
1, d_{t_{(a, b)}} \geq T 1_{t_{(a, b)}}
\end{array} \quad \mathrm{Eq}-4\right.
$$

Where $T 1_{t_{(a, b)}}$ is adaptive thresholding.

\subsection{Modeling background pixels using Gaussian distributions}

To use K Gaussian probability density distributions to constitute the background of each pixel in the image. The pixel $\{a 0, b 0\}$ is defined as a time series.

$\left\{a_{1-\ldots-\ldots-a_{t}}\right\}=\left\{K\left(a_{0}, b_{0} j\right): 1<j<t\right\}$

Where $K$ refers to the image sequence and $a_{j}$ is the intensity value of the pixel $\{a 0, b 0\}$ at time instant $j$.

The background pixels are modeled using Gaussian distribution method. The probability of pixel value within the history values of the pixel is determined as

$\mathrm{f}\left(\mathrm{A}_{\mathrm{t}}\right)=\sum_{j=1}^{K} \omega_{j, t} \mathrm{n}\left(A_{t, \mu_{j, t}} \sum_{\mathrm{j}, \mathrm{t}}\right)$

where $K$ refers to the number of Gaussian distributions used. $\omega_{j, t}$ is the weight parameter that is used to describe which part of the data is described by the $i$ th Gaussian distribution.nis a Gaussian distribution that has two parameters: $\mu_{j, t}$ is the mean of the Gaussian distribution at time $t$ and $\Sigma_{t}$ is the covariance matrix at time instant $t$. Any new pixel value is compared to the existing Gaussian distributions for each frame.If a new pixel is within 2.5 standard deviations of the distribution's 
Bhavana R.Maale et al., International Journal of Science and Advanced Information Technology, 10 (3), May - June 2021,16 - 19

mean, it is said to fit the distribution. If a pixel matches one of the weighted Gaussian distributions, the distribution's mean and variance are modified.

\section{RESULTS}
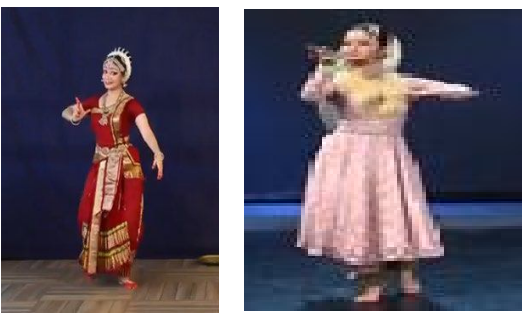

(a)

(b)

Fig 1:Input frames of Kuchipudi(a) and Kathak(b)
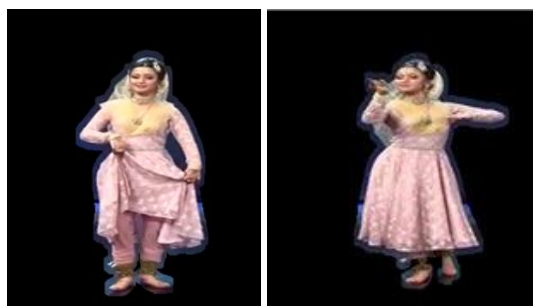

Fig 2:Segmented frames of Kathak dance
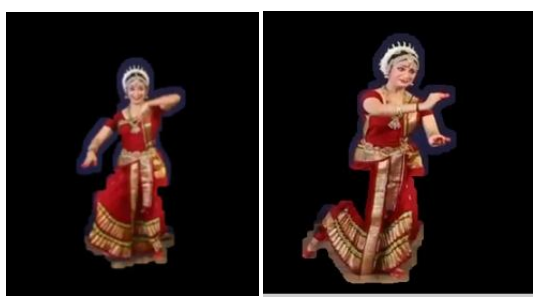

Matlab is used to apply the procedures under investigation. The Gaussian distribution approach is contrasted to the Adaptive Temporal Averaging Method. For two separate videos, kathak and kuchipudi, both methods are used.Each adjustable parameter is expressed as a function of the quality of background subtraction. The F-measure is used to assess the consistency quantitatively. For kathak and kuchipudi, the Gaussian distribution method gives the highest accuracy than the adaptive method, depending on the F-Measure parameter.
Table 1: Comparison Accuracy results of the adaptive and Gaussian distribution method

\begin{tabular}{|l|l|l|l|}
\hline Method & Parameter & \multicolumn{2}{|l|}{ Test Case } \\
\cline { 3 - 4 } & & Kathak & Kuchipudi \\
\hline \multirow{2}{*}{$\begin{array}{l}\text { Adaptive } \\
\text { temporal } \\
\begin{array}{l}\text { averaging } \\
\text { method }\end{array}\end{array}$} & Precision & 0.70 & 0.70 \\
\cline { 2 - 4 } & Recall & 0.89 & 0.89 \\
\cline { 2 - 4 } & F-Measure & 0.68 & 0.79 \\
\hline $\begin{array}{l}\text { Gaussian } \\
\text { distribution } \\
\text { method }\end{array}$ & Precision & 0.75 & 0.75 \\
\cline { 2 - 4 } & Recall & 0.88 & 0.88 \\
\cline { 2 - 4 } & F-Measure & 0.76 & 0.81 \\
\hline
\end{tabular}

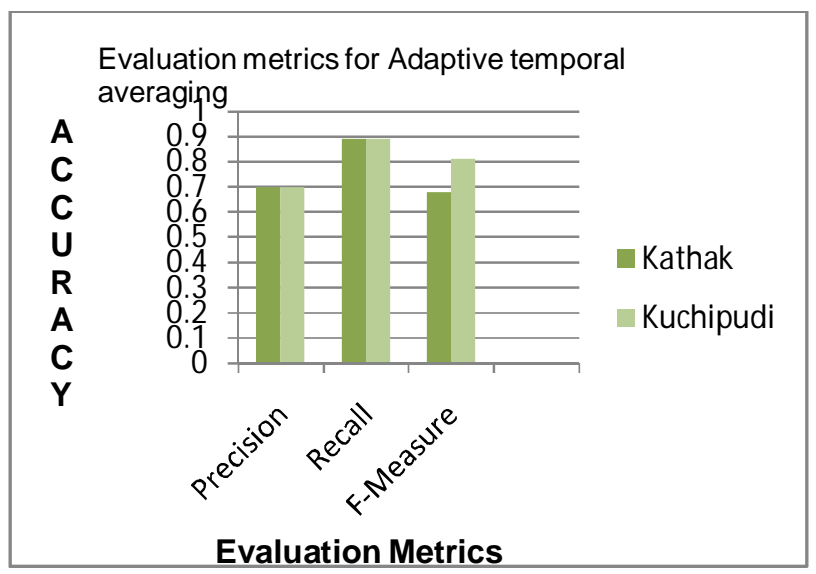

Fig 4: Accuracy graph of the evaluation metrics for Adaptive temporal averaging .

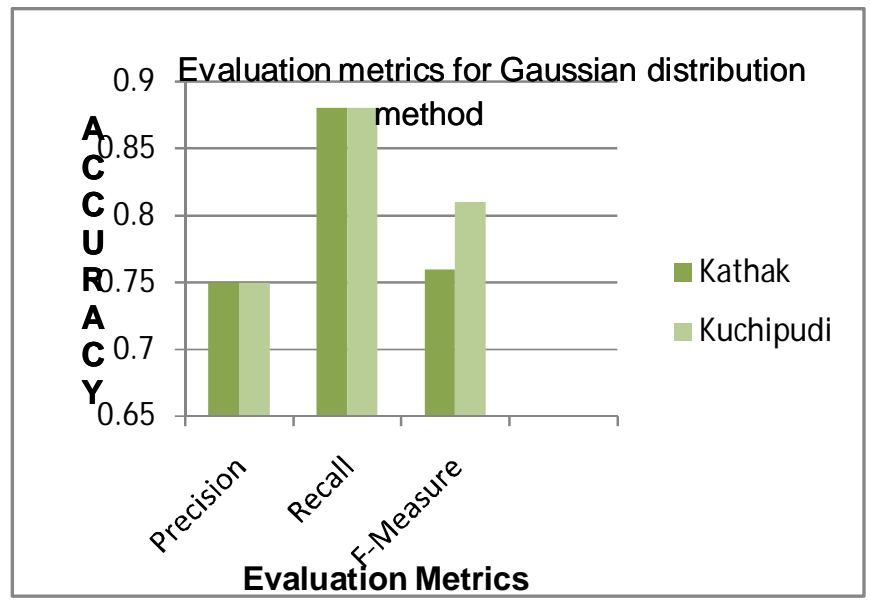

Fig 5 :Accuracy graph of the evaluation metrics for Gaussian distribution method 
Bhavana R.Maale et al., International Journal of Science and Advanced Information Technology, 10 (3), May - June 2021,16 - 19

\section{CONCLUSION}

An adaptive background approach was presented. Gaussian distributions were used to model any other or third pixel in the image in our system. Our process is quicker than the original system, according to the findings. The ability to track moving areas and the amount of noise in the binary motion picture are identical to the original system's properties. Our method can be implemented in a real-world video surveillance framework and run on regular computers due to its speed and robustness. We focused on the algorithm's speed, so some other issues, such as slow learning at first or too quick adaptation of still objects, would need to be addressed in the future.

\section{REFERENCES}

[1] VARGAS, M., TORAL, S. L., BARRERO, F., MILLA, J. M. An enhanced background estimation algorithm for vehicle detection in urban traffic video. In Proceedings of the 11th International IEEE Conference on Intelligent Transportation Systems. Beijing (China), Oct 2008, p. 784790.

[2] MANZANERA, A., RICHEFEU, J. C. A new motion detection algorithm based on sigma-delta background estimation. Pattern Recognition Letters In Advances in Visual Information Processing, Feb 2007, vol. 28, no. 3, p. 320-328.

[3] MCFARLANE, N. J. B., SCHOFIELD, C. P. Segmentation and tracking of piglets in images. Machine Vision and Applications, May 1995, vol. 8, no. 3, p.187-193.

[4] STAUFFER, C., GRIMSON, W. E. L. Adaptive background mixture models for real-time tracking. IEEE InternationalConference on Computer Vision and Pattern Recognition, June 1999, vol. 2, p. 246-252.

[5] BOUWMANS, T., EL BAF, F., VACHON, B. Background modeling using mixture of Gaussians for foreground detection - a survey. Recent Patents on Computer Science, November 2008, vol. 1, no. 3, p. 219237.

[6] DALleY, G., MIGDAL, J., GRIMSON, W. Background subtraction for temporally irregular dynamic textures. In WACV2008. Colorado (USA), January 2008, p. 1-7.

[7] DICKINSON, P., HUNTER, A. Scene modelling using an adaptive mixture of Gaussians in colour and space.
In IEEEConf. on Advanced Video and Signal based Surveillance, AVSS 2005. Como (Italy), September, 2005, p. 64-69.

[8] WANG, H., MILLER, P. Regularized online mixture of Gaussians for background subtraction. In IEEE International Conference on Advanced Video and SignalBased Surveillance, AVSS 2011. Klagenfurt (Austria), September, 2011, p. 249-254.

[9] ELGAMMAL, A., DURAISWAMI, R., HARWOOD, D., DAVIS, L. Background and foreground modeling using nonparametric kernel density estimation for visual surveillance. Proceedings of the IEEE, July 2002, vol. 90, no. 7, p. 1151-1163., MARSHALL, D., MARTIN, R. Merging and splitting eigenspace models. IEEE Trans. Pattern Anal. Mach. Intell., 2000, vol. 22, no. 9, p. 10421049.

[11] HEIKKILA, J., SILVEN, O. A real-time system for monitoring of cyclists and pedestrians. In Proceedings of the Second IEEE Workshop on Visual Surveillance, (VS'99). July 1999, p. 74-81.

[12] FRIEDMAN, N., RUSSELL, S. Image segmentation in video sequences: A probabilistic approach. In Proceedings of the Thirteenth Conference (UAI-1997). San Francisco (USA), 1997,p. 175-181.

[13] HA, D. M., LEE, J. M., KIM, Y. D. Neural-edgebased vehicle detection and traffic parameter extraction. Image and Vision Computing, 2004, vol. 22, no. 11, p. 899-907.

[14] AGARWAL, S., AWAN, A., ROTH, D. Learning to detect objects in images via a sparse, part-based representation. IEEE Trans. On Pattern Analysis and Machine Intelligence, 2004, vol. 26, no. 11,p. 1475-1490.

[15] Mrs BHAVANA R,Dr.SUVARNA NANDYAL. "Segmentation of An Indian Classical Dance Videos using Different Segmentation Methods". International Journal of Innovative Technology and Exploring Engineering (IJITEE) ISSN: 2278-3075, Volume-9 Issue-2, December 2019. 